\title{
Prevalence and Impact of Takotsubo Syndrome in Hospitalizations for Acute Ischemic Stroke
}

\author{
Upenkumar Patel ${ }^{1}$, Rupak Desai ${ }^{2}$, MOHAMMED Faisal Uddin ${ }^{3}$, Hee Kong Fong ${ }^{4}$, Sandeep \\ Singh $^{5}$, Smit Patel $^{6}$, Gautam Kumar ${ }^{7}$, and Rajesh Sachdeva ${ }^{8}$ \\ ${ }^{1}$ Nassau University Medical Center \\ ${ }^{2}$ Atlanta VA Medical Center \\ ${ }^{3}$ Affiliation not available \\ ${ }^{4} \mathrm{UC}$ Davis Department of Medicine \\ ${ }^{5}$ Amsterdam University Medical Centres \\ ${ }^{6}$ Hartford Hospital \\ ${ }^{7}$ Emory University \\ ${ }^{8}$ Morehouse School of Medicine
}

March 12, 2021

\begin{abstract}
Background: Takotsubo Syndrome (TTS) is characterized by acute and reversible left ventricular dysfunction with apical ballooning arising during acute stress situations. Acute Ischemic Stroke (AIS) is one of the known triggers to TTS, however, the impact of TTS on in-hospital outcomes of AIS remains unexplored. Methods: We utilized data from the National Inpatient Sample (2007-2014) and using ICD-9-CM diagnosis codes to identify admissions for AIS with TTS and evaluated the temporal trends, baseline characteristics, in-hospital complications, length of stay (LOS) and all-cause mortality. Survey multivariable logistic regression was used to compute adjusted Odds Ratios (OR and 95\% confidence intervals (CI). Results: An estimated $2,242(0.4 \%)$ TTS cases were identified among AIS hospitalizations $(\mathrm{N}=43,92,471)$. The frequency of TTS was higher in elderly, whites and females $(82.2 \%)$. After adjustment for confounders, TTS incidence in AIS was associated with higher odds of in-hospital complications including cardiogenic shock (OR8.84, CI:4.07-19.17, p<0.001), cardiac arrest (OR3.17, CI:1.57-6.42, $\mathrm{p}=0.001$ ), and venous thromboembolism (OR1.68, CI:1.14-2.47, $\mathrm{p}=0.008$ ). Moreover, AIS hospitalizations with TTS showed higher odds of developing respiratory failure (OR3.13, CI:2.42-4.05, p<0.001) and required mechanical ventilation/intubation (OR4.09, CI:3.14-5.32, p<0.001) more frequently compared to non-TTS cohort. The AIS-TTS cohort had longer LOS (8.59 vs 5.22 days) and the mortality was doubled $(10.2 \%$ vs $5.1 \% \mathrm{p}<0.001)$ compared to those without TTS. Conclusion: The prevalence of TTS in AIS remained 20 times higher than the general inpatient population and it predisposed AIS patients to worse inpatient outcomes. Further studies are needed to evaluate the impact of TTS on long-term outcomes in AIS.
\end{abstract}

\section{Introduction}

Takotsubo Syndrome (TTS) is a relatively reversible heart condition characterized by left ventricular (LV) dysfunction, apical ballooning with abnormally elevated cardiac biomarkers and ECG changes in the absence of any obstructive coronary artery disease [1]. The precise cause of this cardiomyopathy is not known but possible explanations include catecholamine surge during stress, ischemia-mediated stunning of the myocardium due to microvascular spasm, and myocarditis $[2,3]$. The TTS has been one of the wellknown complications following subarachnoid hemorrhage, occurring due to increased catecholamine release following intracranial hemorrhage $[4,5]$. The overall prognosis of TTS most of the time is favorable, however, certain clinical conditions carry higher risk and may have poorer outcomes [6]. Therefore, recognition and 
management of such clinical conditions that might predispose the patients to TTS is of utmost importance and should be an integral part of the management [3].

With the rising prevalence of TTS in various clinical conditions, multiple studies have reported TTS trends and outcomes in various neurological and non-neurological clinical conditions [7-10]. The acute cerebral events including acute ischemic stroke (AIS) are associated with catecholamine surge and hence, can lead to TTS. However, limited data exist on the prevalence and outcomes of TTS in AIS. This study aims at evaluating the US nationwide estimate of the burden and the differences in the outcomes of TTS and non-TTS in the inpatient AIS population.

\section{Methods}

We queried the National Inpatient Sample (NIS) (2007 to 2014) a nationally weighted data of all hospital discharges in the United States to describe the frequency of various complications in AIS patients with TTS and also to determine the predictors of all-cause in-hospital mortality. The NIS is a part of the Healthcare Cost and Utilization Project (HCUP) funded by the Agency for Healthcare Research and Quality (AHRQ) [11]. This data consists of a sample from $20 \%$ of total hospitalizations from nonfederal US community hospitals representing $95 \%$ of the general population in the US. The NIS contains de-identified data; hence, our study was exempt from an institutional review board authorization.

We used International Classification of Diseases, 9th Revision, Clinical Modification (ICD-9-CM) to identify primary admissions for AIS (433.01, 433.11, 433.21, 433.31, 433.81, 433.91, 434.01, 434.11, 434.91 and 436) and then using ICD-9 CM code 429.83 admissions with TTS as a secondary discharge diagnoses were filtered.

The primary outcome of our study was to assess the prevalence, trends and all-cause inpatient mortality in AIS cohort with TTS vs. without TTS. Secondary outcomes included frequency of in-hospital complications such as cardiogenic shock, venous thromboembolism, respiratory failure, need for mechanical ventilation and healthcare resource utilization [discharge disposition, mean length of stay (LOS) and hospital charges] among AIS-TTS cohort as compared to AIS- non-TTS cohort.

We utilized designated weight values to produce nationally representative estimates and survey multivariable logistic regression models were performed after adjusting patients and hospital-level confounders to estimate the odds and 95\% confidence intervals (CI) of in-hospital mortality and other complications in AIS cohort with TTS as compared to without TTS. We used SPSS v22 (IBM Corp., Armonk, NY) to perform all statistical analyses.

\section{Results}

From 2007-14, 43,92,471 hospitalizations with AIS were identified, of which, 2242 (0.4\%) had TTS. An increasing trend in prevalence of TTS was seen from 2007 with the highest number of cases recorded in 2014 $(\mathrm{n}=1081)$. Higher proportion of AIS-TTS cohort consisted of elderly (.65) (69.8\%), white (77.5\%) females $(82.2 \%)$.

AIS-TTS cohort showed higher prevalence of comorbidities including congestive heart failure $(37.5 \%$ vs $14 \%$; $\mathrm{p}<0.001)$, coronary atherosclerosis $(32.2 \%$ vs $25.7 \% ; \mathrm{p}<0.001)$, previous myocardial infarction $(10.4 \%$ vs $6.4 \%$; $<<0.001$ ), valvular disease (14.1\% vs $10 \%$; $<<0.001$ ), alcohol abuse $(6.5 \%$ vs $3.9 \% ; \mathrm{p}<0.001)$, deficiency anemias $(20.2 \%$ vs $11.5 \%$; $<<0.001)$, rheumatoid arthritis/collagen vascular diseases $(4.2 \%$ vs $2.4 \% ; \mathrm{p}<0.001$, chronic pulmonary disease ( $20.8 \%$ vs $14.9 \%$; $<0.001)$, coagulopathy $(6.2 \%$ vs $4.8 \% ; \mathrm{p}<0.001)$, fluid and electrolytes disorders (37.4\% vs $19.9 \%$; $<<0.001)$ and hypothyroidism (14.3\% vs $12.5 \% ; \mathrm{p}<0.001)$ as compared to AIS patients without TTS.

All-cause in-hospital mortality in AIS patients with TTS was double than patients without TTS (10.2\% vs $5.1 \% ; \mathrm{p}<0.001)$. Moreover, AIS hospitalizations were associated with higher odds of cardiogenic shock (OR 8.84, CI:4.07-19.17; $\mathrm{p}<0.001$ ), cardiac arrest (OR 3.17, CI:1.57-6.42; $\mathrm{p}=0.001$ ), respiratory failure (OR 3.13, CI:2.42-4.05; $\mathrm{p}<0.001$ ), venous thromboembolism (OR 1.68, CI:1.14-2.47; $\mathrm{p}=0.008$ ), and need for intubation/mechanical ventilation (OR 4.09, CI:3.14-5.32; $\mathrm{p}<0.001$ ) among AIS patients with TTS. 
Mean LOS was higher among AIS-TTS cohort as compared to non-TTS cohort (8.59 \pm 10.03 vs 5.22 \pm 6.24 ; $\mathrm{p}<0.001$ ). AIS patients with TTS required chronic care and step down to the Skilled Nursing Facility (SNF), Intermediate Care Facility (ICF) more frequently compared to non-TTS patients ( $53.8 \%$ vs $42.9 \%$ ). In addition, mean hospital charges were higher among AIS-TTS cohort $(\$ 85,854$ vs $\$ 37,924)$.

\section{Discussion}

To the best of our knowledge, this is the first study demonstrating the prevalence, temporal trends and impact of TTS in patients with AIS. In this nationally representative sample of hospitalizations with AIS, we demonstrate an estimated $0.4 \%$ of TTS cases from 2007-14 which were associated with higher all-cause mortality, adverse hospital-related complications and longer LOS as compared to AIS patients without TTS. This rate is nearly 20 times higher than the TTS rate reported in the general inpatient population (0.02\%). Previous analyses studying TTS in AIS have either been single-center studies or case reports [12-15].

There has been an increasing trend in the prevalence of TTS with AIS from 2007-2014, with the highest number of cases recorded during $2014(\mathrm{~N}=1081)$. The highest proportion of TTS cases among AIS were recorded among elderly $>65$ years $(69.8 \%)$ and female $(82.2 \%)$ patients. A study by Jung et al. of 23 AIS patients reported similar findings of increasing rates of TTS among the elderly with a mean age of $70.7 \pm 13.9$ years consisting predominantly of females $(73.9 \%)$ [16]. Female predominance was seen in another study by Templin et al [17]. Consistent with our study findings, a study by Murugiah et al. reported white patients representing almost $90.2 \%$ of the total TTS population [18].

The baseline characteristics and comorbidities could define prognosis in AIS patients with TTS. We observed a significantly higher prevalence of congestive heart failure, chronic pulmonary disease, coagulopathy, fluid and electrolytes disorders, hypothyroidism, obesity, previous myocardial infarction, depression and coronary atherosclerosis among AIS patients with TTS. In one study by Brinjikji et al. reported the higher prevalence of chronic cardiovascular morbidities among TTS patients [19]. These data could help better risk stratify patients at risk of TTS with AIS.

In our study, we observed higher odds of developing adverse events during AIS hospitalizations with TTS. These included cardiogenic shock $(\mathrm{OR}=8.84)$, cardiac arrest $(\mathrm{OR}=3.17)$, and venous thromboembolism $(\mathrm{OR}=1.68)$. Moreover, the AIS-TTS cohort was at higher risk of developing respiratory failure $(\mathrm{OR}=3.13)$ and requiring mechanical ventilation/intubation $(\mathrm{OR}=4.09)$ more frequently. Increased risk of cardiovascular complications in AIS-TTS cohort could be due to higher proportion of elderly patients with underlying chronic medical illness. Similar findings were observed in a study by Brinjikji et al, in which higher odds of complications were seen among elderly patients with underlying chronic cardiovascular risk factors [19].

TTS occurrence in AIS also had a worse impact on healthcare resource utilization. We observed frequent step down of AIS-TTS cohort to more chronic care centers including SNF, ICF etc. In addition, we observed an extended hospital stay of TTS cohort ( $8.59 \pm 10.03$ vs $5.22 \pm 6.24)$ compared to non-TTS cohort. This could be attributed to increased risk of complications requiring longer stay and more advanced care. Prolonged hospital stay among AIS-TTS cohort was also associated with more than two times higher mean total hospital charges $(\$ 85,854$ vs $\$ 37,924)$. This further signifies the importance of identifying AIS patients early to prevent complications and associated economic repercussions.

The all-cause inpatient mortality observed in AIS patients with TTS was double $(10.2 \%$ vs $5.1 \%)$ than non-TTS cohort. In addition to the added burden of TTS-related complications, this could be explained by the higher prevalence of chronic comorbidities, which increases risk of in-hospital adverse events among AIS patients with TTS. In a prospective observational study, higher mortality risk along with adverse events was observed in older age groups [20].

\section{Limitations}

Our study should be reviewed with certain limitations to our analysis. Since we used admission-associated data rather than patient-level data we depended on administrative diagnosis codes. Therefore, the outcomes among the patients who were discharged cannot be defined due to limited information of follow-up. We were 
also unable to account for unmeasured confounders that could be associated with increased risk of TTS in AIS patients and the complications occurring during hospital stay. Data on medication records and follow-up were not available. Despite these limitations, the benefit of using a large, multi-center, representative sample affords a truly national perspective and preserves the validity of associations reported.

\section{Conclusion}

The prevalence of TTS among admissions for AIS was $0.4 \%$, which is nearly 20 times higher than the TTS rate reported in the general inpatient population. The higher prevalence of TTS was seen in elderly, white and female patients with AIS. The TTS occurrence was associated with higher in-hospital mortality, cardiovascular complications and healthcare resource utilization. Further studies are warranted to evaluate the association and potential long-term impact of TTS in AIS patients.

\section{Acknowledgement: None}

\section{References}

[1] M. Madhavan, A. Prasad. Proposed Mayo Clinic criteria for the diagnosis of Tako-Tsubo cardiomyopathy and long-term prognosis, Herz. 35 (2010) 240-243. 10.1007/s00059-010-3339-x

[2] K.A. Bybee, T. Kara, A. Prasad, et al. Systematic review: transient left ventricular apical ballooning: a syndrome that mimics ST-segment elevation myocardial infarction, Ann. Intern. Med. 141 (2004) 858-865. 10.7326/0003-4819-141-11-200412070-00010

[3] F. Pelliccia, G. Parodi, C. Greco, et al. Comorbidities frequency in Takotsubo syndrome: an international collaborative systematic review including 1109 patients, Am. J. Med. 128 (2015) 654.e611-659. 10.1016/j.amjmed.2015.01.016

[4] V.H. Lee, H.M. Connolly, J.R. Fulgham, E.M. Manno, R.D. Brown, Jr., E.F. Wijdicks. Tako-tsubo cardiomyopathy in aneurysmal subarachnoid hemorrhage: an underappreciated ventricular dysfunction, $J$. Neurosurg. 105 (2006) 264-270. 10.3171/jns.2006.105.2.264

[5] M. Shimada, J.D. Rose. Takotsubo cardiomyopathy secondary to intracranial hemorrhage, Int. J. Emerg. Med. 7 (2014) 33-33. 10.1186/s12245-014-0033-4

[6] R. Ono, L.M. Falcão. Takotsubo cardiomyopathy systematic review: Pathophysiologic process, clinical presentation and diagnostic approach to Takotsubo cardiomyopathy, Int. J. Cardiol . 209 (2016) 196-205. 10.1016/j.ijcard.2016.02.012

[7] R. Desai, S.A. Abbas, H.K. Fong, et al. Burden and impact of takotsubo syndrome in myasthenic crisis: A national inpatient perspective on the under-recognized but potentially fatal association,Int. J. Cardiol. 299 (2020) 63-66. 10.1016/j.ijcard.2019.09.054

[8] R. Desai, S.A. Abbas, H. Goyal, et al. Frequency of Takotsubo Cardiomyopathy in Adult Patients Receiving Chemotherapy (from a 5-Year Nationwide Inpatient Study), Am. J. Cardiol . 123 (2019) 667-673. 10.1016/j.amjcard.2018.11.006

[9] R. Desai, A. Desai, S.A. Abbas, et al. National prevalence, trends and outcomes of takotsubo syndrome in hospitalizations with prior history of mediastinal/intrathoracic cancer and radiation therapy,Int. J. Cardiol. 309 (2020) 14-18. 10.1016/j.ijcard.2020.02.036

[10] R. Desai, S. Singh, U. Patel, et al. Frequency of takotsubo cardiomyopathy in epilepsy-related hospitalizations among adults and its impact on in-hospital outcomes: A national standpoint, Int. J. Cardiol. 299 (2020) 67-70. 10.1016/j.ijcard.2019.07.034

[11] HCUP-US NIS Overview. https://www.hcup-us.ahrq.gov/nisoverview.jsp. Accessed September 1, 2018.

[12] C. Blanc, M. Zeller, Y. Cottin, et al. Takotsubo Cardiomyopathy Following Acute Cerebral Events, Eur. Neurol. 74 (2015) 163-168. 10.1159/000440717 
[13] I. Porto, R. Della Bona, A. Leo, et al. Stress cardiomyopathy (tako-tsubo) triggered by nervous system diseases: a systematic review of the reported cases, Int. J. Cardiol. 167 (2013) 2441-2448. 10.1016/j.ijcard.2013.01.031

[14] F. Santoro, E. Carapelle, S.I. Cieza Ortiz, et al. Potential links between neurological disease and TakoTsubo cardiomyopathy: a literature review, Int. J. Cardiol. 168 (2013) 688-691. 10.1016/j.ijcard.2013.03.093

[15] S. Yoshimura, K. Toyoda, T. Ohara, et al. Takotsubo cardiomyopathy in acute ischemic stroke, Ann. Neurol. 64 (2008) 547-554. 10.1002/ana.21459

[16] J.M. Jung, J.G. Kim, J.B. Kim, et al. Takotsubo-Like Myocardial Dysfunction in Ischemic Stroke: A Hospital-Based Registry and Systematic Literature Review, Stroke 47 (2016) 2729-2736. 10.1161/strokeaha.116.014304

[17] C. Templin, J.R. Ghadri, J. Diekmann, et al. Clinical Features and Outcomes of Takotsubo (Stress) Cardiomyopathy, N. Engl. J. Med. 373 (2015) 929-938. 10.1056/NEJMoa1406761

[18] K. Murugiah, Y. Wang, N.R. Desai, et al. Trends in Short- and Long-Term Outcomes for Takotsubo Cardiomyopathy Among Medicare Fee-for-Service Beneficiaries, 2007 to 2012, JACC Heart Fail. 4 (2016) 197-205. 10.1016/j.jchf.2015.09.013

[19] W. Brinjikji, A.M. El-Sayed, S. Salka. In-hospital mortality among patients with takotsubo cardiomyopathy: a study of the National Inpatient Sample 2008 to 2009, Am. Heart J. 164 (2012) 215-221. 10.1016/j.ahj.2012.04.010

[20] R. Citro, F. Rigo, M. Previtali, et al. Differences in clinical features and in-hospital outcomes of older adults with tako-tsubo cardiomyopathy, J. Am. Geriatr. Soc. 60 (2012) 93-98. 10.1111/j.15325415.2011.03730.x

Figure legends

Figure 1. Trends in Takotsubo Syndrome Hospitalizations

A. Trends in Takotsubo Syndrome per 1 Million Hospitalizations for Acute Ischemic Stroke

B. Trends in Takotsubo Syndrome per 1 Million Hospitalizations for Acute Ischemic Stroke Stratified by Stroke

C. Trends in Takotsubo Syndrome per 1 Million Hospitalizations for Acute Ischemic Stroke Stratified by Age

D. Trends in Takotsubo Syndrome per 1 Million Hospitalizations for Acute Ischemic Stroke Stratified by Race/Ethnicity

Cover Letter

Date: $03 / 06 / 2021$

Dr Charles Young, Editor-in-Chief, International Journal of Clinical Practice

Re: Short Communication: Prevalence and Impact of Takotsubo Syndrome in Hospitalizations for Acute Ischemic Stroke

Dear Dr. Young,

We herewith submit a Short Communication entitled "Prevalence and Impact of Takotsubo Syndrome in Hospitalizations for Acute Ischemic Stroke" for possible publication in your esteemed journal after your kind review.

Upcoming reports suggest Acute Ischemic Stroke is one of the known triggers to TTS. The acute cerebral events including acute ischemic stroke (AIS) are associated with catecholamine surge and hence, can lead to TTS. However, limited data exist on the prevalence and outcomes of TTS in AIS. Furthermore, the impact 
of TTS on in-hospital outcomes of AIS remains unexplored. Using the National Inpatient Sample (20072014) databases and relevant ICD-9 CM codes, we identified AIS and TTS-related adult hospitalizations and assessed the frequency of TTS in AIS patients and its impact on disposition of patients and healthcare resource utilization in the United States.

We hope the editorial board and reviewers will find the content suitable for International Journal of Clinical Practice. This manuscript, as submitted or its essence in another version is not under consideration for publication elsewhere and will not be published elsewhere while under consideration by International Journal of Clinical Practice. The authors have no commercial associations or sources of support that might pose a conflict of interest. All authors have made substantive contributions to the manuscript, and all authors endorse the data and conclusions.

We have no relationships with industry to disclose.

Thank you for your consideration.

Yours sincerely,

Rupak Desai, MBBS

Division of Cardiology

Atlanta Veterans Affairs Medical Center, 1670 Clairmont Rd, Decatur, GA 30033

Email:rdesa30@emory.eduPhone: (404) 321-6111 ext. 7359

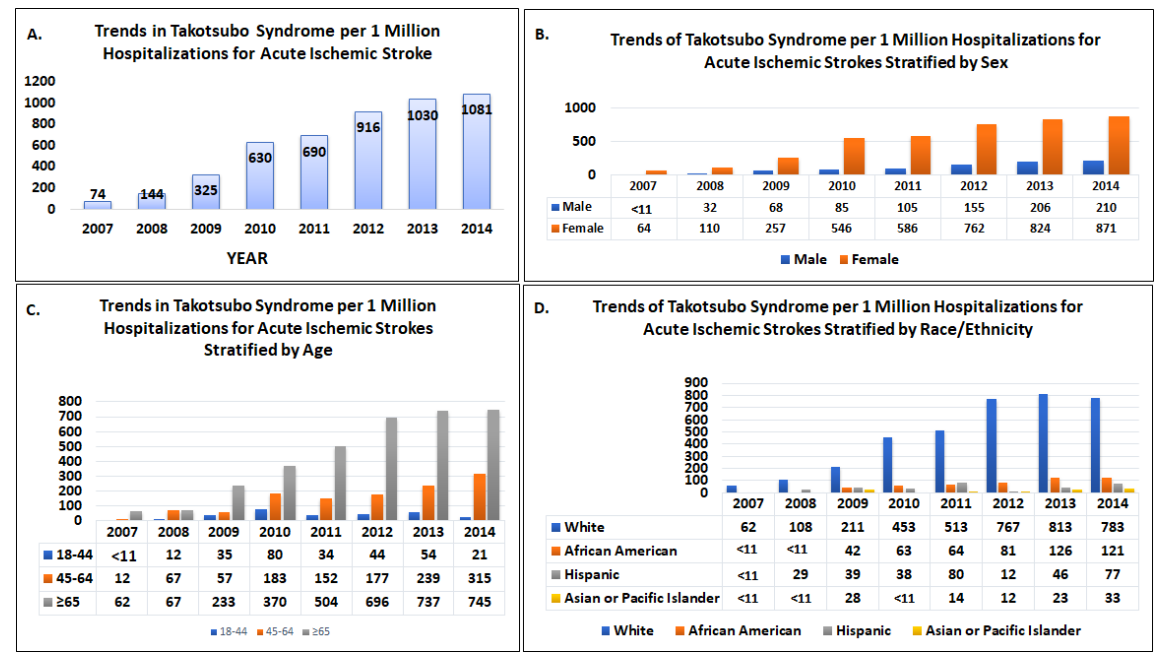

\section{Hosted file}

Table 1.pdf available at https://authorea.com/users/401133/articles/513333-prevalence-andimpact-of-takotsubo-syndrome-in-hospitalizations-for-acute-ischemic-stroke

\section{Hosted file}

Table 2.pdf available at https://authorea.com/users/401133/articles/513333-prevalence-andimpact-of-takotsubo-syndrome-in-hospitalizations-for-acute-ischemic-stroke 